\title{
Catching a Grown-Up Starfish Planetary Nebula: I. Morpho-Kinematical study of PC 22
}

\author{
L. Sabin ${ }^{1 \star}$, M.A. Gómez-Muñoz ${ }^{1}$, M.A. Guerrero ${ }^{2}$, S. Zavala ${ }^{3,4}$, G. Ramos-Larios ${ }^{5}$, \\ R. Vázquez ${ }^{1}$, L. Corral ${ }^{5}$, M.W. Blanco Cárdenas ${ }^{1}$, P.F. Guillén ${ }^{1}$, L. Olguín ${ }^{6}$, C. Morisset ${ }^{7}$, \\ S. Navarro ${ }^{5}$ \\ ${ }^{1}$ Instituto de Astronomía, Universidad Nacional Autónoma de México, Apdo. Postal 877, 22800 Ensenada, B.C., Mexico \\ ${ }^{2}$ Instituto de Astrofísica de Andalucía, IAA-CSIC, C/ Glorieta de la Astronomía s/n, 18008 Granada, Spain \\ ${ }^{3}$ Instituto Tecnológico de Ensenada, Blvd Tecnológico No. 150, 22780 Ensenada, B. C., Mexico \\ ${ }^{4}$ Instituto de Estudios Avanzados de Baja California, A. C., Blvd Tte. Azueta 147, Edif. Matematiké Planta Baja, 22800 Ensenada, B. C., Mexico \\ ${ }^{5}$ Instituto de Astronomía y Meteorología, Av. Vallarta No. 2602, Col. Arcos Vallarta, C.P. 44130 Guadalajara, Jalisco, Mexico \\ ${ }^{6}$ Dpto. de Investigación en Física, Universidad de Sonora, Blvd. Rosales-Colosio, Ed. 3H, 83190, Hermosillo, Sonora, Mexico \\ ${ }^{7}$ Instituto de Astronomía, Universidad Nacional Autónoma de México, Apdo. Postal 70264, Mexico D. F. 04510, Mexico
}

Last updated $2016 \mathrm{xx} x \mathrm{x}$; in original form $2016 \mathrm{xx}$ xx

\begin{abstract}
We present the first part of an investigation on the planetary nebula (PN) PC 22 which focuses on the use of deep imaging and high resolution echelle spectroscopy to perform a detailed morpho-kinematical analysis. PC 22 is revealed to be a multipolar PN emitting predominantly in [O III] and displaying multiple non-symmetric outflows. Its central region is found to be also particularly inhomogeneous with a series of low ionization structures (knots) located on the path of the outflows. The morpho-kinematical model obtained with SHAPE indicates that i) the de-projected velocities of the outflows are rather large, $\geqslant 100 \mathrm{~km} \mathrm{~s}^{-1}$, while the central region has expansion velocities in the range $\sim 25$ to $\sim 45 \mathrm{~km} \mathrm{~s}^{-1}$ following the "Wilson effect", ii) the majority of the measured structures share similar inclination, $\simeq 100^{\circ}$, i.e. they are coplanar, and iii) all outflows and lobes are coeval (within the uncertainties). All these results make us to suggest that PC 22 is an evolved starfish PN. We propose that the mechanism responsible for the morphology of PC 22 consists of a wind-shell interaction, where the fast post-AGB wind flows through a filamentary AGB shell with some large voids.
\end{abstract}

Key words: (ISM:) planetary nebulae: individual: PC 22 - ISM: jets and outflows — ISM: kinematics and dynamics

\section{INTRODUCTION}

Planetary Nebulae (PNe) are well known for displaying different degrees of morphological complexity probing their, often intense, internal dynamical activity (see the imaging catalogues by, Parker et al. 2006, 2016; Sahai et al. 2011 and Sabin et al. 2014). Hence, the presence of outflows, jets, rings or knots each indicate particular physical processes whose origins are not always well constrained. By establishing with accuracy the morpho-kinematics and chemical characteristics of $\mathrm{PNe}$, one can expect to better understand their formation history. Such multi-approach analyses have been performed to study in great detail various PNe such as NGC 3132 (Monteiro et al. 2000), IPHASX J194359.5+170901 (Corradi et al. 2011), K 4-55 and Kn 26 (Guerrero et al. 1996, 2013, respectively), M 2-48 (López-Martín et al. 2002), ETHOS 1 (Miszalski et al.

* E-mail:1sabin@astro.unam.mx (LS)
2011), and NGC 6309 (Vázquez et al. 2008). With this aim in mind, we present in this article a detailed investigation of a peculiar PN, PC 22 (PN G051.0-04.5) located at $\alpha_{2000}=19: 42: 03.50$, $\delta_{2000}=+13: 50: 37.33$.

Discovered by Apriamashvili (1959) and later catalogued by Peimbert \& Costero (1961), this object, has not been subject of any extensive targeted study so far. Manchado et al. (1996) classified PC 22 as an elliptical planetary nebula with "internal structures" (no description of these were made). Although no binary system has been detected, Soker (1997) predicted a high probability for the progenitor to have gone through a common envelope with a sub-stellar companion based on this morphology. Except for photometric measurements realized at various wavelengths we found no other relevant piece of information about the PN.

The distance to PC 22 seems to be relatively well constrained. Tajitsu \& Tamura (1998) obtained a distance of $5.5 \mathrm{kpc}$ for this object by fitting a blackbody to the flux of the four IRAS bands, 
whereas Giammanco et al. (2011) estimated a distance of $4.0 \pm 0.5$ $\mathrm{kpc}$ based on the distance-extinction method built with the first IPHAS data release ${ }^{1}$. More recently Frew et al. (2016), using a new $\mathrm{H} \alpha$ surface brightness-radius relation, derived a mean distance of $5.27 \pm 1.52 \mathrm{kpc}$ adopting $\log (\mathrm{S}(\mathrm{H} \alpha))=-2.77 \pm 0.10$ $\operatorname{erg~} \mathrm{cm}^{-2} \mathrm{~s}^{-1} \mathrm{sr}^{-1}$.

In this paper we present new deep morpho-kinematical observations which reveal a wealth of information on PC 22 and assert its physical structure. The article is organized as the following: the observations are described in $\S 2$; the results obtained from each observing technique are described in $\$ 3$ and this includes our proposed kinematical model. Finally we conclude our investigation presenting the main findings in $\$ 4$ followed by the discussion and concluding remarks in $\$ 5$. This is the first paper on a series which focuses on the morpho-kinematical analysis of PC 22. We will present a comprehensive chemical analysis in a forthcoming paper (Sabin et al. in preparation).

\section{OBSERVATIONS}

\subsection{ALFOSC optical imaging}

Direct narrow-band images of PC 22 were obtained on 2009 September 4 using the Alhambra Faint Object Spectrograph and Camera (ALFOSC) at the $2.5 \mathrm{~m}$ Nordic Optical Telescope (NOT) at the Roque de los Muchachos Observatory (ORM, La Palma, Spain). The detector was a $2048 \times 2048 \mathrm{EEV} \mathrm{CCD}(13.5 \mu \mathrm{m}$ pixel size) with a plate scale of $0 . \prime 184 \mathrm{pix}^{-1}$ providing a field of view of $6 ! 3 \times 6 ! 3$. The $\mathrm{H} \alpha \quad\left(\lambda_{c}=6567 \AA\right.$, FWHM $\left.=8 \AA\right)$, [N II] $\left(\lambda_{c}=6588 \AA, \mathrm{FWHM}=9 \AA\right)$, and [O III] $\left(\lambda_{c}=5007 \AA\right.$, FWHM $=30 \AA$ ) filters were used to acquire two $900 \mathrm{~s}$ images in each filter. The images were processed using standard IRAF routines. A mean spatial resolution of $00^{\prime \prime} 6$ was achieved, as determined from the FWHM of stars in the field.

\subsection{MES long-slit high resolution optical spectroscopy}

Long-slit high dispersion optical spectra of PC 22 were acquired with the Manchester Echelle Spectrograph (MES, Meaburn et al. 2003) mounted on the $2.12 \mathrm{~m}$ telescope at the Observatorio Astronómico Nacional, San Pedro Mártir (OAN-SPM, Mexico). A first set of data was obtained on 2015 August 14-16. At this time, a $2048 \times 2048$ pixels E2V CCD with a pixel size of $13.5 \mu \mathrm{m} \mathrm{pixel}^{-1}$ was used as detector. The fixed slit length is 6.5 and we set the slit width to $150 \mu \mathrm{m}\left(1{ }^{\prime \prime} 9\right)$. We used $2 \times 2$ and $4 \times 4$ binnings leading to spatial scales of $0 . " 351$ pixel $^{-1}$ and 0.702 pixel $^{-1}$, respectively. The different spectra were taken with exposures of $1200 \mathrm{~s}$ and 1800 s through two filters: an $\mathrm{H} \alpha$ filter with $\Delta \lambda=90 \AA$ to isolate the 87 th order $\left(0.05\right.$ and $0.1 \AA$ pixel $^{-1}$ spectral scale for the $2 \times 2$ binning and $4 \times 4$ binning, respectively) and an [O III] $5007 \AA$ filter with $\Delta \lambda=50 \AA$ to isolate the 114th order $(0.043$ and 0.086 $\AA$ pixel $^{-1}$ spectral scale for the $2 \times 2$ binning and $4 \times 4$ binning, respectively). We mostly used the latter filter based on the dominant [O III] emission in the PN (as seen in the images, see below). Additional spectra were taken on 2016 February 25 using the same settings.

All the spectra were bias and flat corrected, and wavelength calibrated using standard IRAF routines for long-slit spectroscopy.

1 Since then a new photometric calibration has been performed by Barentsen et al. (2014).
The wavelength-calibration was performed with a ThAr arc lamp to an accuracy of $\pm 1 \mathrm{~km} \mathrm{~s}^{-1}$. The FWHM of the arc lamp emission lines, $\simeq 12 \pm 1 \mathrm{~km} \mathrm{~s}^{-1}$, provides an estimate of the spectral resolution.

\subsection{ESPRESSO multi-order optical echelle spectroscopy}

Multi-order echelle spectroscopic observations were performed on 2014 May 29 using ESPRESSO, an Echelle spectrograph mounted on the $2.12 \mathrm{~m}$ telescope at the OAN-SPM (Mexico). The E2V CCD detector described above was used. The wavelength range covered was 3900-7290 $\AA$ distributed through 27 orders with a spectral scale $\sim 0.1 \AA \mathrm{pixel}^{-1}$ at $5000 \AA$. The slit was positioned at an angle of $47^{\circ}$ passing through the central star. No binning was applied and the exposure time was set to $1800 \mathrm{~s}$. The data reduction, which includes bias removal and flat fielding correction, was performed with MIDAS (Grosbol 1989). Two-dimensional spectra of each order was then extracted and wavelength calibrated with a ThAr arc lamp using IRAF routines. We note the absolute wavelength calibration of the spectral orders covering the [O III] $\lambda 5007$ and [Ar V] $\lambda 7006$ emission lines was not optimal. No flux calibration was performed. Each order was then analyzed separately and no attempt to bring them together was made.

\section{RESULTS}

\subsection{Morphology}

Our deep and high resolution $\mathrm{H} \alpha$, [N II] and [O III] images of PC 22 unveil a detailed picture of a complex multipolar morphology. The "elliptical" structure once believed to form the whole nebula is actually the central part of a PN with multiple outflows (Fig. 1). We identified six large lobes or outflows (numbered 1-6 in Fig. 1) and three secondary lobes or outflows closer to the central elliptical region (numbered 7-9 in Fig. 1). The [O III] 5007 ̊ nebular emission is significantly bright and shows an extension of $\sim 1.5 \times 00^{\prime} .9$ including the tip of the faintest components presented in Fig. 2 and Fig. 3. The high level of [O III] 5007 $\AA$ emission is somewhat peculiar as it extends up to the outermost regions of the PN and is not confined to the inner parts of the nebula as one would expect (i.e. there is no stratification in the emission lines distribution). Such behavior, however, is not unique and it has been noted in other PNe such as NGC 6309 (Rubio et al. 2015) and NGC 6905 (Cuesta et al. 1993).

The faint aforementioned structures are under the form of filaments (in the south for example) and weak outflows which are best seen in the contrast-enhanced image shown in Fig. 3. Three arc-like patterns, labelled "A, B and C" can be associated to the tips of three different outflows detailed below.

The $\mathrm{H} \alpha$ and [O III] images indicate a bright and slightly collimated group of ejecta along the SE-NW direction (hereafter Axis 1), with the NW outflow appearing to be "broken" by the emergence of a new one whose much dimer rim is mostly seen in [O III] (Fig. 3- label "C"). This effect is not symmetric, i.e. the structure "C" does not have a clear counterpart in the SE outflow. In the N-S direction (hereafter Axis 2), the presence of a bright inner section immediately followed by a much fainter one at larger distances (seen in [O III]) could indicate the occurrence of two different outflows running approximately along the same direction. The presence of these new structures is supported by the arc-like patterns "A" and "B" observed in Fig. 3. Once again, there is not a clear, symmetric counterpart to the northern outflows. Fig. 3 also 


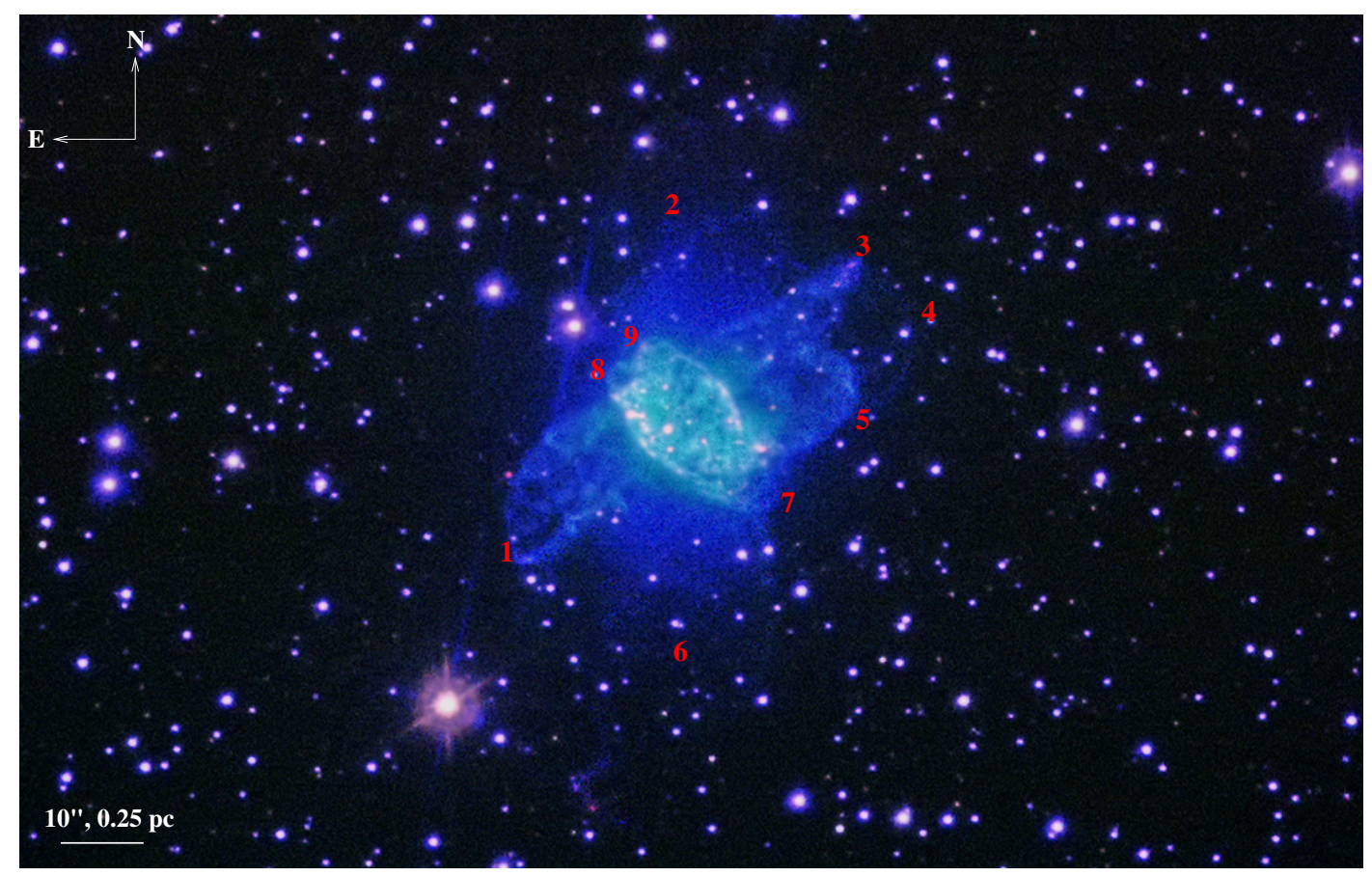

Figure 1. Nordic Optical Telescope RGB picture of $\mathrm{PC} 22(\mathrm{R}=\mathrm{H} \alpha, \mathrm{G}=[\mathrm{N}$ II $], \mathrm{B}=[\mathrm{O}$ III $]$ ). The image reveals a non-homogeneous central elliptical structure and several ejections reaching distances up to $\sim 50^{\prime \prime}$ from the central star. The numbers indicate the different outflows identified in this nebula.
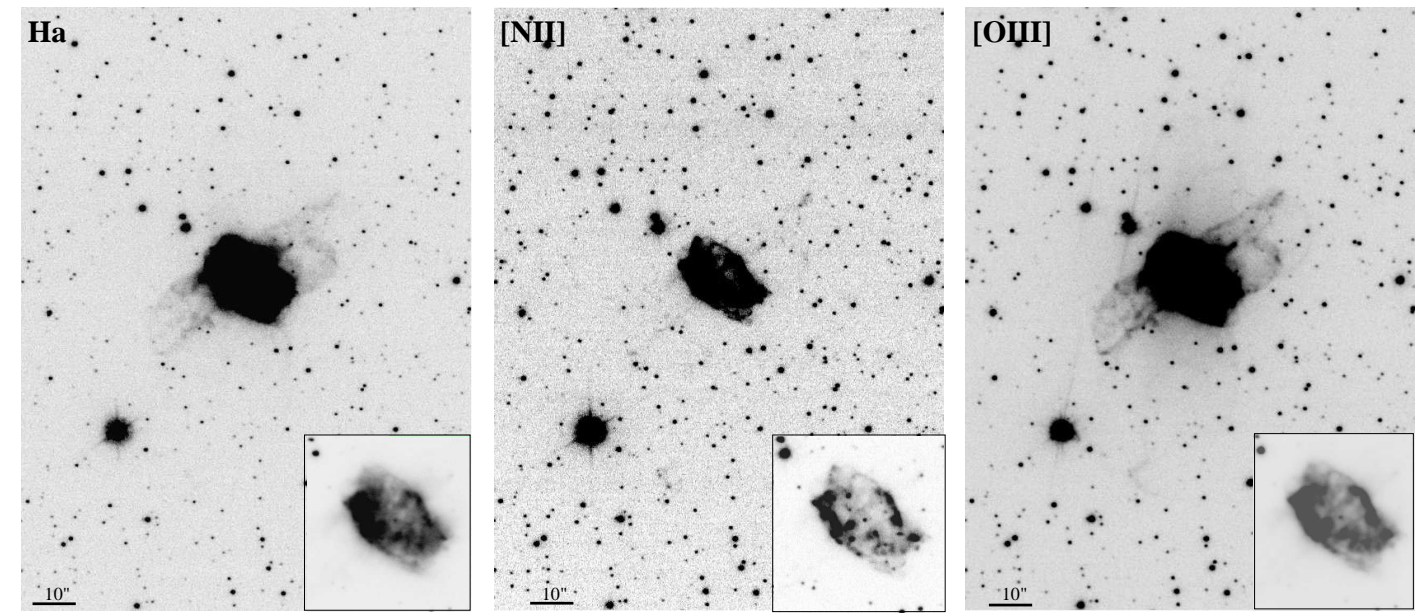

Figure 2. Detailed gray-scale rendering of the $\mathrm{H} \alpha$, [N II], and [O III] NOT images. In the largest images $\left(\mathrm{FoV} \simeq 1.7^{\prime} \times 2.2^{\prime}\right)$ the gray-scale levels are chosen to unveil the faintest and most diffused structures of the PN, while saturating the central regions. A number of outflows and filaments are seen mostly in [O III]. The inset on each figure shows a zoom of the elliptical central region that underlines its inhomogeneity with various high density clumps best seen in [N II] distributed around the central star.

shows that the ejecta in the Axis 2 are wider, less collimated, less defined and generally fainter than those in Axis 1 . There is also a slight difference in the dominating emission lines (and hence in the ionization factor). Whereas [O III] emission prevails in Axis 2, a combination of $\mathrm{H} \alpha$ and $[\mathrm{N}$ II] emissions are prevalent in Axis 1. A more exhaustive analysis of these two regions, from a chemical point of view, will be presented in the second part of this investigation (Sabin et al. in preparation).

The central region of PC 22 has a size of 13 ". $5 \times 23^{\prime \prime} 9$ and a position angle (P.A.) on the plane of the sky of $49^{\circ}$. It shows a notable inhomogeneous morphology in all emission lines (Fig. 2).
The "clumpy"/knotty nature of this region is better seen in [N II], which is a known tracer of low ionization structures compared to the higher ionization [O III] emission line (Corradi et al. 1996). We produced different line ratio images, namely $(\mathrm{H} \alpha+[\mathrm{N} \mathrm{II}]) /[\mathrm{O} I I I]$ and $[\mathrm{N} \mathrm{II}] /[\mathrm{O} I I I]$, to check for the presence of such patterns (Fig. 4). No new structures were revealed besides those already seen. In terms of spatial distribution, the clumps described above are seen inside and (mostly) at the edges of the elliptical ring, i.e., the inhomogeneities are concentrated in the central region. More precisely the main over-density positions are coincident with the base of the ejecta related to the Axis 1, suggesting the irregular brightening or 


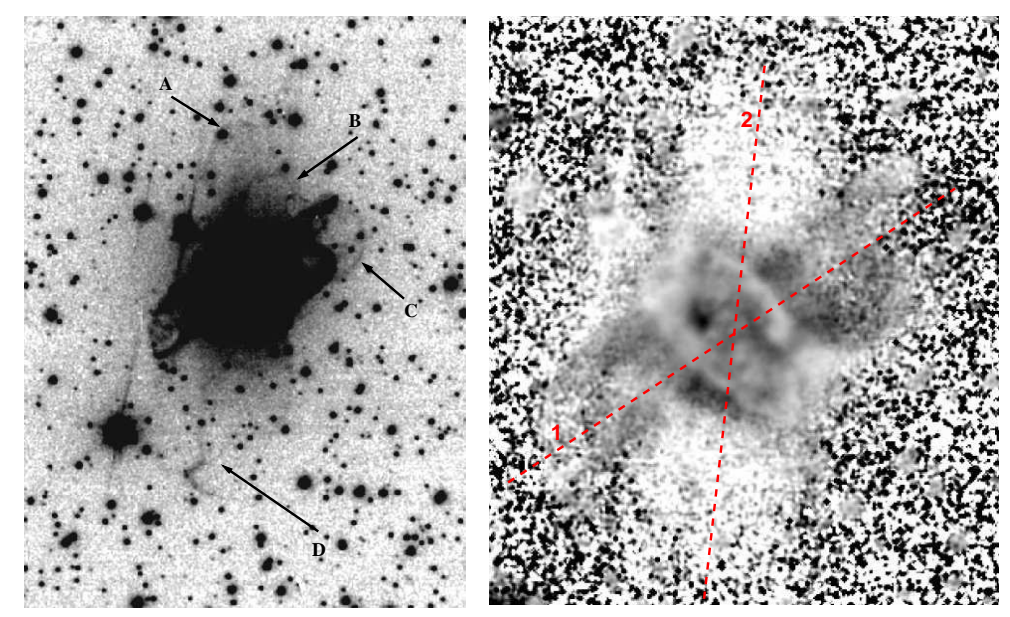

Figure 3. Left panel: $[\mathrm{O} \mathrm{III}]$ image $\left(\mathrm{FoV} \simeq 1.7^{\prime} \times 2.2^{\prime}\right)$ with grey-scale levels chosen to highlight the faintest patterns such as the arcs or outflows tips labelled $\mathrm{A}, \mathrm{B}$, and $\mathrm{C}$ and the filamentary structure $\mathrm{D}$. Right panel: Gaussian filtered ratio map $(\mathrm{H} \alpha+[\mathrm{N} \mathrm{II}]) /[\mathrm{O} \mathrm{III}]\left(\mathrm{FoV} \simeq 1^{\prime} \times 1.2^{\prime}\right)$ where the lighter zones imply dominant [O III] emission over $\mathrm{H} \alpha+[\mathrm{N}$ II $]$. The two dotted lines indicate the two main regions or axis showing different ionization degrees.

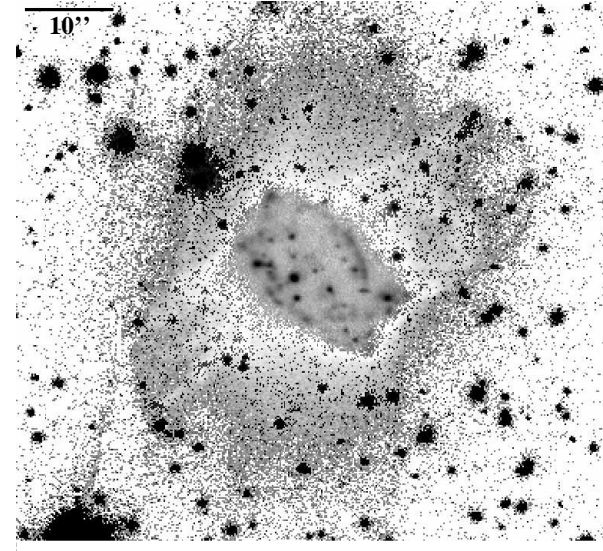

Figure 4. $[\mathrm{N} \mathrm{II}] /[\mathrm{O} \mathrm{III}]$ ratio map underlying the bright knots and clumpy medium in the central part of PC 22.

ionization of material following the passage of a collimated outflows. Such interaction is not unusual and has been described by Vázquez et al. (2000) in M2-48.

The morphological analysis of PC 22 unveils a much more complex object than initially described by an elliptical morphology. The PN shows multiple non symmetric ejecta with two main differing degrees of ionization whose "waist" seems to be shaped by the interaction with the different outflows.

\subsection{Kinematics}

\subsubsection{Morpho-kinematical modelling of MES data with SHAPE}

The large set of slit positions (see Fig. 5 and Tab. 1) provides a unique coverage of PC 22 to carry out a comprehensive kinematical analysis. The observations used in the following will be those taken in the [O III] line. Using the seven positions passing through the central star, we derived a mean observed systemic velocity $\bar{V}_{o b s}$ of $28.1 \mathrm{~km} \mathrm{~s}^{-1}$, which corresponds to an LSR mean value of 36.4 $\mathrm{km} \mathrm{s}^{-1}$.

Although we were able to secure a good mapping of the PN, the overall higher brightness of the central region and the lobes
Table 1. Description of the slits used for the MES observations. "Offset CSPN" corresponds to the slit offset from the central star.

\begin{tabular}{|c|c|c|c|c|}
\hline Slit & $\begin{array}{l}\text { P.A. } \\
\left({ }^{\circ}\right)\end{array}$ & Filter & $\begin{array}{c}\text { Offset CSPN } \\
\left({ }^{\prime \prime}\right)\end{array}$ & $\begin{array}{l}\mathrm{T}_{\exp } \\
(\mathrm{s})\end{array}$ \\
\hline A & 5 & [O III $]$ & 0 & 1800 \\
\hline B & 28 & [O III] & 0 & 1800 \\
\hline $\mathrm{C}$ & 49 & {$[\mathrm{O} \mathrm{III}], \mathrm{H} \alpha$} & 0 & 1200 \\
\hline $\mathrm{D}$ & 49 & [O III] & 13.3 & 1200 \\
\hline $\mathrm{E}$ & 49 & [O III] & 13.0 & 1200 \\
\hline $\mathrm{F}$ & 75 & [O III $]$ & 0 & 1200 \\
\hline G & 100 & [O III] & 0 & 1200 \\
\hline $\mathrm{H}$ & 137 & {$[\mathrm{O} \mathrm{III}], \mathrm{H} \alpha$} & 0 & 1200 \\
\hline I & 137 & [O III] & 10.0 & 1200 \\
\hline J & 137 & [O III $]$ & 8.0 & 1200 \\
\hline K & 166 & [O III] & 0 & 1200 \\
\hline $\mathrm{L}$ & 128 & [O III] & 2.1 & 1800 \\
\hline M & 114 & [O III] & 3.4 & 1800 \\
\hline
\end{tabular}

Table 2. Kinematical characteristics (i.e. velocities and age) for eight different structural components obtained from our best fitting model constructed with SHAPE (see Fig.7). Velocities are relative to the calculated systemic of $36.4 \mathrm{~km} \mathrm{~s}^{-1}$ and $\theta$ is the angular radius measured from the central star. We note that the de-projected velocity $\left(\mathrm{V}_{\text {dep }}\right)$ for structure 7 is likely to be "affected" by the presence of other velocity field components from its surrounding.

\begin{tabular}{ccrccc}
\hline $\begin{array}{c}\text { Structures } \\
\text { number }\end{array}$ & $\begin{array}{c}\mathrm{V}_{\text {rad }} \\
\left(\mathrm{km} \mathrm{s}^{-1}\right)\end{array}$ & $\begin{array}{c}\theta_{\text {dep }} \\
\left({ }^{\prime \prime}\right)\end{array}$ & $\begin{array}{c}i \\
\left({ }^{\circ}\right)\end{array}$ & $\begin{array}{c}\mathrm{V}_{\text {dep }} \\
\left(\mathrm{km} \mathrm{s}^{-1}\right)\end{array}$ & $\begin{array}{c}\mathrm{t}_{\text {kin }} \\
(\mathrm{yrs})\end{array}$ \\
\hline 1 & $-9 \pm 3$ & 12.1 & 83 & 131 & $2300 \pm 700$ \\
2 & $-40 \pm 7$ & 13.2 & 74 & 140 & $2400 \pm 800$ \\
3 & $-9 \pm 4$ & 8.6 & 100 & 56 & $3800 \pm 1400$ \\
4 & $21 \pm 4$ & 8.3 & 100 & 59 & $3500 \pm 1300$ \\
5 & $23 \pm 3$ & 15.1 & 103 & 101 & $3700 \pm 1200$ \\
6 & $26 \pm 5$ & 23.0 & 100 & 155 & $3700 \pm 1100$ \\
7 & $12 \pm 3$ & 5.4 & 100 & $33:$ & $4100 \pm 2000$ \\
8 & $-7 \pm 3$ & 6.5 & 100 & 48 & $3400 \pm 1400$ \\
\hline
\end{tabular}



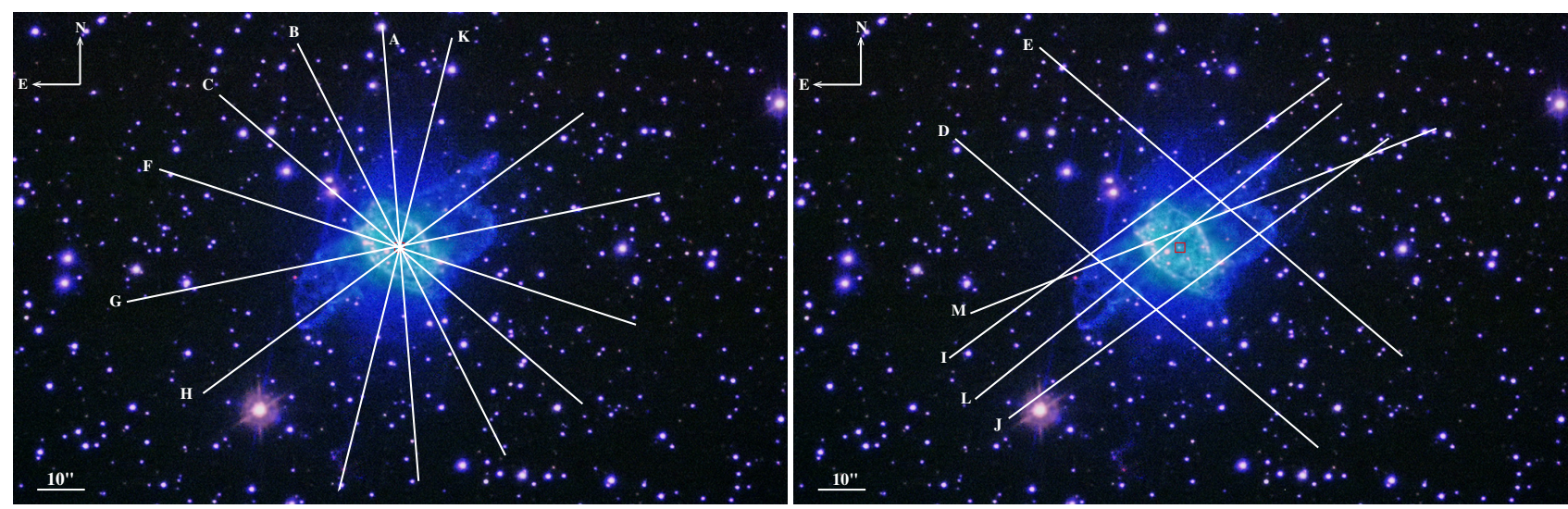

Figure 5. Slit positions used for the MES observations superimposed on the optical RGB picture of PC 22. On the left panel we present all those passing through the central star (red square) and the remaining are shown on the right panel. The thirteen positions are meant to provide the best coverage of the PN for the kinematic analysis.

corresponding to the Axis 1 were the only structures to actually appear clearly in the spectra, with the other components being much fainter. The upper panels of Fig. 6 show the resulting spectra or position-velocity (PV) maps, passing through the short and long axis of the main internal structure $\left(\mathrm{PA}=49^{\circ}\right.$ : slit $\mathrm{C}$ and $\mathrm{PA}=137^{\circ}$ : slit $\mathrm{H}$, respectively) and the bright lobes in Axis 1 at $\mathrm{PA}=100^{\circ}$ corresponding to the slit $\mathrm{G}$. In all three cases we note the irregular shape of the inner regions which corresponds to the presence of compact knots. The remaining observed PV maps are presented in the appendix A, Fig. A1 and A2.

We subsequently used the morpho-kinematical tool SHAPE (Steffen et al. 2011) to model all the PV diagrams and obtain a complete view of the (kinematical and morphological) structure of PC 22. Due to the inhomogeneous nature of the central part, we concentrated only on the representation and modelling of the basic velocity patterns corresponding to an equatorial ring and several bipolar outflows. Therefore, one will observe a difference between the actual (thicker) observed spectra and the narrower synthetic ones (see the lower panels of Fig. 6). The non-inclusion of the knots/clumps does not hamper the overall kinematical analysis. We should keep in mind that the spectra are not treated independently i.e. a change in the position/morphology or the velocity of one spectrum will affect the position/morphology or the velocity of the adjacent one(s). The full set of PV diagrams are shown in Fig. 6 and the appendices A1 and A2. The subsequent SHAPE reconstruction of the PN is shown in Fig. 7. Table 2 summarizes the data for the brightest (and best defined) components. In this case we derived information for the two northern "bumps" (\#1,\#2), the southern small outflow (\#5), the four extensions of the internal ellipsoid (\#3,4,7 and 8) and finally one of the western outflows (\#6). In all cases the velocities were measured at the tip of the structures. For each we calculated the radial velocities $\left(\mathrm{V}_{\text {rad }}\right)$ and their respective errors, the de-projected angular radii $\left(\theta_{d e p}\right)$, the inclinations $(i)$, de-projected velocities $\left(\mathrm{V}_{d e p}\right)$ and kinematical ages $\left(\mathrm{t}_{k i n}\right)$. The errors on $i$ and $\mathrm{V}_{\text {dep }}$ were derived empirically solely based on our model and we estimated a mean error on the inclination of $\sim 5^{\circ}$ and a mean error on $\mathrm{V}_{\text {dep }}$ of $\sim 3 \mathrm{~km} \mathrm{~s}^{-1}$. Those are the typical values above which we observed a notable variation in the PV maps. The inclinations found indicate that most of the structures are nearly lying in the plane of the sky. The deduced de-projected velocities allow us to distinguish two groups: the medium velocities structures, from $\sim 33$ to $59 \mathrm{~km} \mathrm{~s}^{-1}$ (although we note that the

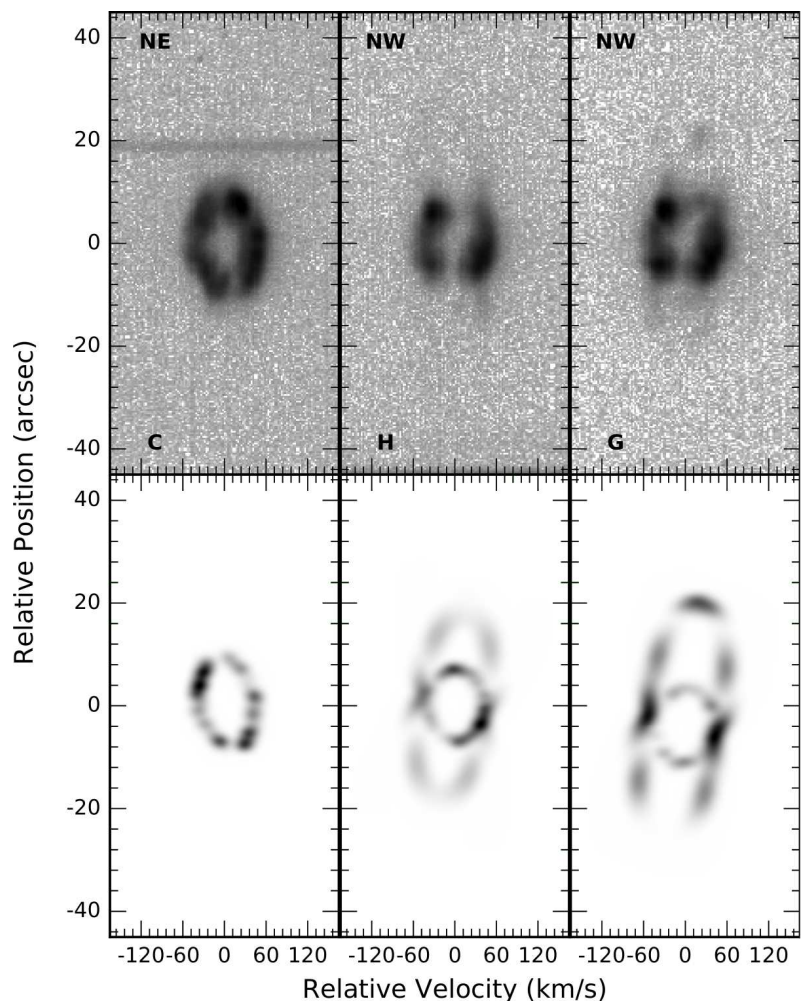

Figure 6. Top: MES [O III] position-velocity (PV) plots of three of the main slit positions with higher signal-to-noise level, namely slit positions C, G, and $\mathrm{H}$ (see Tab. 1 and Fig. 5, where the $\mathrm{C}$ and $\mathrm{H}$ slits go across the nebula center along P.A. $49^{\circ}$ and $137^{\circ}$, respectively, and slit $\mathrm{H}$ is offset form the central star and goes along P.A. $100^{\circ}$. The binning factor is $2 \times 2$ in all images. Bottom: SHAPE kinematical modelling of these lines for the model assumed in the text.

former value is very likely affected by nearby structures), which are related to the central region, and the high velocities (jets-like) ones, above $\sim 100 \mathrm{~km} \mathrm{~s}^{-1}$ related to the "bumps" and larger outflows.

The kinematical age of these components can be derived using 


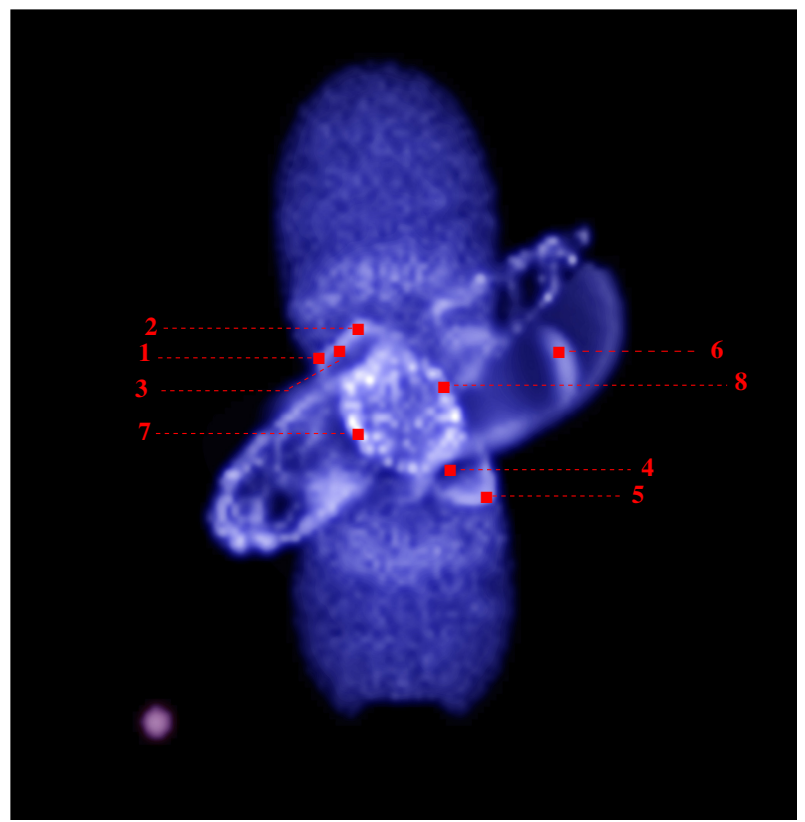

Figure 7. SHAPE reconstruction of PC 22 based on all PV diagrams. The numbers indicate the structures of reference for the different kinematical calculations (see table 2).

the relationship:

$$
t_{k i n}=\frac{4744 * d(k p c) * \theta\left(^{\prime \prime}\right)}{V_{d e p}(k m / s)} .
$$

We can clearly see two groups: components \#1 and \#2, related to the northern "bumps" or small outflows, have a mean age of $2300 \pm 800 \mathrm{yr}$, whereas the other components have a mean age of $3600 \pm 1300 \mathrm{yr}^{2}$. The elliptical region, if interpreted as a ring, has a kinematical age of $\sim 4500$ yrs. Within the errors, the different components are coeval, but we notice that the interaction of the outflows with the circumstellar medium would reduce their expansion velocity (thus increasing their kinematical age). The kinematical analysis completes the morphological one, pointing towards a PN mostly shaped by the interaction of high-velocity non-symmetric winds, the zone of the interaction being the central region which as a consequence is highly inhomogeneous. Most of the events (e.g. outflows launching) also appear to have occurred at approximately the same time in the life of the not so old PC 22. This will give us an important constraint on the evolutionary history of this PN (see section 5).

\subsubsection{ESPRESSO}

The inspection of the ESPRESSO echelle spectrum of the central region of PC 22 reveals a notable number of emission lines that present blue and red components (Fig. 8). These can be interpreted as the approaching and receding walls of the central structural component, respectively, and therefore they provide information on its internal kinematics. A list of these lines is reported in Table 3. The radial velocities associated with each line has been determined by

2 Component \#7, which suffers from the presence of adjacent knots that add velocity components not necessarily related to this part of the shell, has not been taken into account in this average.
Table 3. Kinematical analysis based on the different line splits observed in the ESPRESSO Echelle spectra at $\mathrm{PA}=47^{\circ}$. The values were derived through a Gaussian fit of the lines. For both the blue- and redshifted lines we present: the observed velocities $\left(\mathrm{V}_{o b s}\right)$, the observed data corrected from LSR $\left(\mathrm{V}_{L S R}\right)$ and the velocities relative to the systemic $\left(\mathrm{V}_{\text {rel }}\right)$. Note that, due to difficulties in the absolute wavelength calibration of the spectral orders covering the $[\mathrm{O} \mathrm{III}]$ and $[\mathrm{Ar} \mathrm{V}]$ emission lines, only the observed velocities are quoted for these lines.

\begin{tabular}{lccccccc}
\hline \multirow{2}{*}{ Line ID } & \multicolumn{3}{c}{ Blueshifted lines $(\mathrm{km} / \mathrm{s})$} & & \multicolumn{3}{c}{ Redshifted lines $(\mathrm{km} / \mathrm{s})$} \\
\cline { 2 - 3 } & $\mathrm{V}_{\text {obs }}$ & $\mathrm{V}_{\text {LSR }}$ & $\mathrm{V}_{\text {rel }}$ & & $\mathrm{V}_{\text {obs }}$ & $\mathrm{V}_{L S R}$ & $\mathrm{~V}_{\text {rel }}$ \\
\hline [O III] 5007 & -21.9 & - & - & & 55.2 & - & - \\
He II 5411 & -30.7 & 6.6 & -29.8 & & 37.7 & 74.9 & 38.6 \\
He I 5876 & -43.8 & -6.5 & -42.9 & & 31.4 & 68.7 & 32.3 \\
[S III] 6312 & -33.2 & 4.1 & -32.3 & & 38.9 & 76.2 & 39.8 \\
[N II] 6548 & -37.2 & 0.1 & -36.3 & & 40.9 & 78.3 & 41.8 \\
He II 6560 & -34.0 & 3.3 & -33.1 & & 29.3 & 66.6 & 30.2 \\
H $\alpha$ 6563 & -39.4 & -2.1 & -38.5 & & 30.2 & 67.5 & 31.1 \\
[N II] 6583 & -44.8 & -7.5 & -43.9 & & 33.9 & 71.3 & 34.9 \\
[S II] 6716 & -42.1 & -4.8 & -41.2 & & 42.9 & 80.3 & 43.9 \\
[S II] 6731 & -39.3 & -1.9 & -38.4 & & 41.6 & 78.9 & 42.5 \\
[Ar V] 7005 & -41.9 & - & - & 12.0 & - & - \\
[Ar III] 7135 & -37.5 & -0.2 & -36.6 & 42.8 & 80.2 & 43.8 \\
\hline
\end{tabular}

Table 4. Ionization potential vs expansion velocity for the line with clear splitting seen in PC 22

\begin{tabular}{lccc}
\hline Ion & $\begin{array}{c}\text { Line } \\
(\AA)\end{array}$ & $\begin{array}{c}\text { Ionization potential } \\
(\mathrm{eV})\end{array}$ & $\begin{array}{c}\mathrm{V}_{\text {exp }} \\
\left(\mathrm{km} \mathrm{s}^{-1}\right)\end{array}$ \\
\hline$[\mathrm{S} \mathrm{II}]$ & 6716 & 10.36 & 42.5 \\
{$[\mathrm{~S} \mathrm{II}]$} & 6731 & 10.36 & 40.4 \\
$\mathrm{H} \alpha$ & 6563 & 13.59 & 34.8 \\
{$[\mathrm{~N} \mathrm{II}]$} & 6548 & 14.53 & 39.1 \\
{$[\mathrm{~N} \mathrm{II}]$} & 6583 & 14.53 & 39.4 \\
{$[\mathrm{~S} \mathrm{III}]$} & 6312 & 23.34 & 36.1 \\
$\mathrm{He}$ I & 5876 & 24.59 & 37.6 \\
{$[\mathrm{Ar}$ III $]$} & 7135 & 27.63 & 40.2 \\
{$[\mathrm{O} \mathrm{III}]$} & 5007 & 35.12 & 38.5 \\
$\mathrm{He}$ II & 5411 & 54.42 & 34.2 \\
He II & 6560 & 54.42 & 31.6 \\
{$[$ Ar V] } & 7006 & 59.81 & 26.9 \\
\hline
\end{tabular}

fitting Gaussian curves to each component to derive their wavelengths. We estimated that the maximum error on the line measurement is $\sim 6 \mathrm{~km} \mathrm{~s}^{-1}$. Table 3 reports the observed $\left(V_{\text {obs }}\right)$, LSR corrected $\left(V_{\mathrm{LSR}}\right)$, and relative (from systemic velocity, $\left.V_{\text {rel }}\right)$ velocities for both blueshifted and redshifted components. The systemic velocity, as derived from the ESPRESSO data, is $36.4 \mathrm{~km} \mathrm{~s}^{-1}$, which is consistent with that derived from the MES data.

The expansion velocities are different for different lines. We note that there is a trend for the expansion velocity to increase as the ionization potential of the species increases, as revealed by the linear fit to the data (Table 4 and Fig. 9). Since species with higher ionization potential are closer to the CSPN, it means there is a negative velocity gradient as material is located further away from the CSPN. This is the so-called "Wilson effect" (Wilson 1950), which describes the decrease of the line separation (or expansion velocity) with an increase of the ionization potential in PNe. 


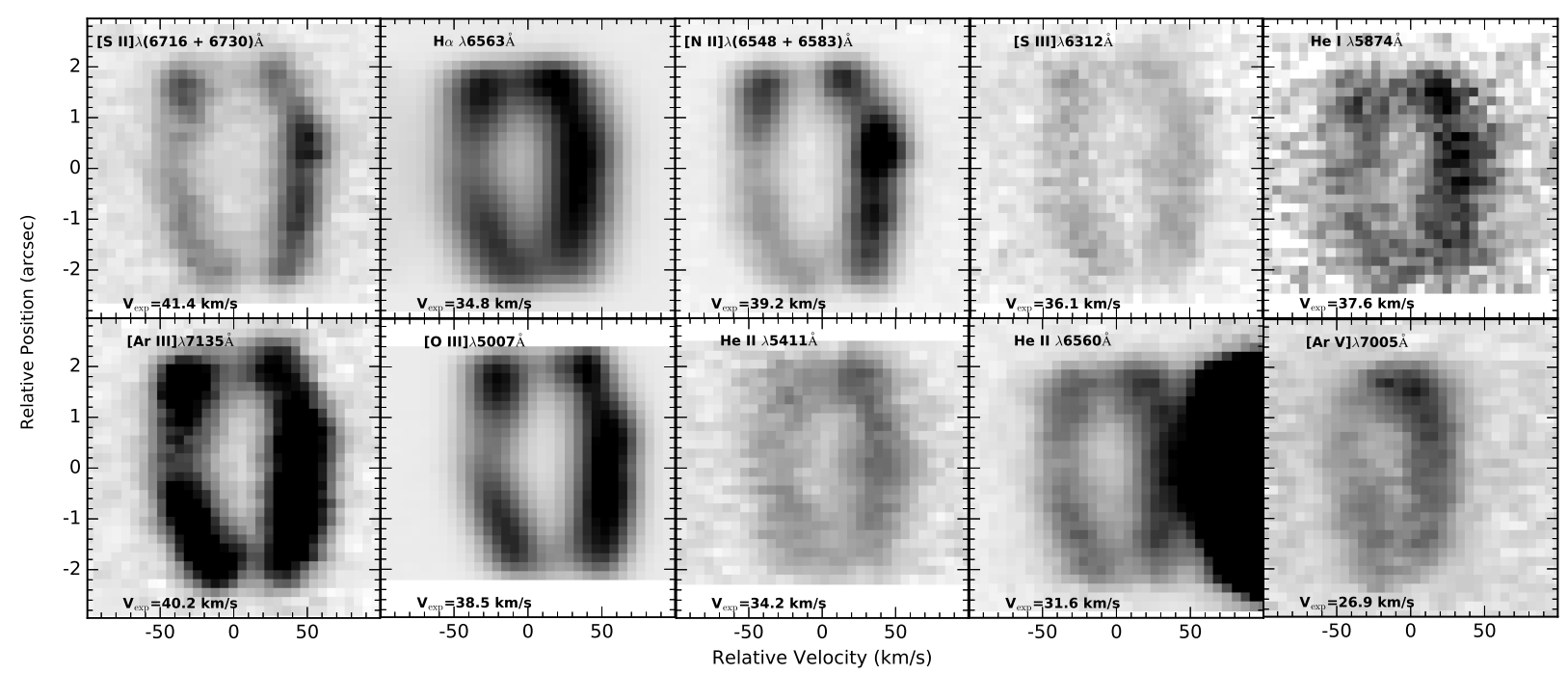

Figure 8. Position-Velocity (PV) diagrams resulting of the analysis of twelve well defined emission lines from the ESPRESSO echelle spectra of PC 22. All lines show a split and the corresponding expansion velocities are quoted. The PVs are arranged in terms of increasing potential ionization level so as to visually follow the change in $\mathrm{V}_{\text {exp }}$. We notice that the [S II] and [N II] emission lines have been added to increase the signal-to-noise ratio. As for the He II (6560 $\AA$ ) line, the dark area on the right hand-side corresponds to the much brighter contiguous $\mathrm{H} \alpha$ line.

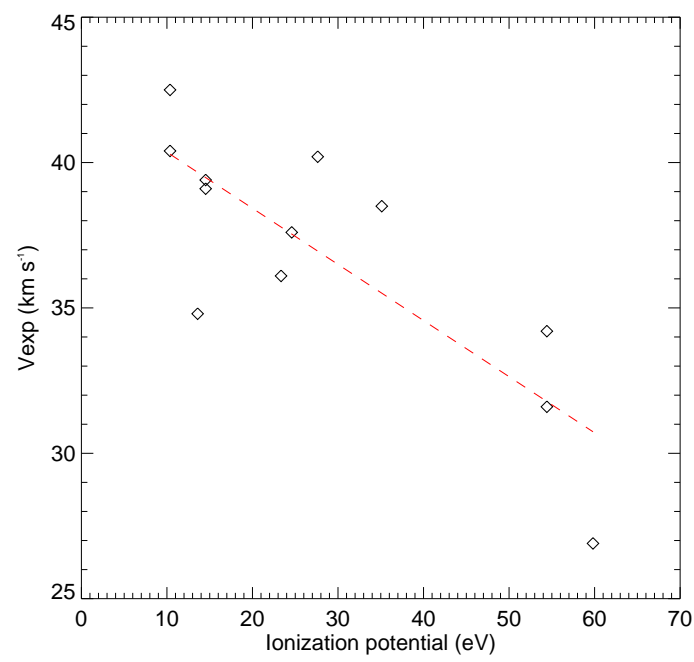

Figure 9. Graph render of Table 4 with the red dotted line representing the linear fit.

\section{MAIN FINDINGS}

The main results of the morpho-kinematical study of PC 22 presented here are the following:

- The PN, principally emitting in [O III], is not elliptical as previously stated, but multipolar with several sets of non-symmetric outflows showing different degrees of brightness, collimation and ionization.

- Most (if not all) over-density and low-ionization structures (e.g., the $[\mathrm{N} \mathrm{II}]$ bright) are concentrated in the elliptical central part of the nebula and are spatially related to the passage of the aforementioned outflows.

- The morpho-kinematical model obtained with SHAPE reveals information on the different structural components of PC 22. Hence:

- The de-projected velocities of the outflows and bumps are rather large $\left(\geqslant 100 \mathrm{~km} \mathrm{~s}^{-1}\right)$ compared to that of the central elliptical region $\left(\sim 30-60 \mathrm{~km} \mathrm{~s}^{-1}\right)$.

- All the structures share similar inclination around $100^{\circ}$ (slightly less for the structures \#1 and \#2 in Fig.7), i.e., nearly all the ejections can be considered as coplanar and close to the plane of the sky.

- The kinematical age calculations are also well consistent for all the analysed outflows with a mean of $3600 \pm 1300 \mathrm{yrs}$, but for structures \#1 and \#2, which seem to be slightly "younger" (2300 \pm 800 yrs). This may indicate either that structural features at the elliptical central region have been accelerated, or that the outflows are been slowed down (see, for instance, the ejecta in the born-again PNe A 30 and A 78, Fang et al. 2014). This is a tantalizing possibility, but at this moment we conclude that all ejections in PC 22 should be considered to be coeval, owing to the uncertainties on the age calculation.

- The ESPRESSO echelle data obtained for twelve emission lines presenting blue- and red-shifted components allowed us to derive their expansion velocities. This varies from 26.9 to $42.5 \mathrm{~km} \mathrm{~s}^{-1}$ and tends to abide by the "Wilson effect" as we noted a decrease of the expansion velocity with an increase of the ionization potential.

\section{DISCUSSION AND CONCLUSION}

The combination of the morphological and kinematical analysis indicates that PC 22 belongs to the category of so-called starfish PNe 
at a late evolutionary stage. Starfish nebulae were introduced by Sahai (2000) to describe Hen 2-47 and M 1-37, two young PNe investigated during an $\mathrm{H} \alpha$ imaging survey with the Hubble Space Telescope. Later, the proto-PN IRAS 19024+0044 (Sahai et al. 2005) was added to this group. All three objects present the same morphological characteristics: they show multiple well defined and fast ( 100-200 $\mathrm{km} \mathrm{s}^{-1}$ ) collimated lobes with approximately the same sizes which would indicate that they have emerged at the same time and an equatorial waist or ring. Our claim that PC 22 is very likely an evolved version of the aforementioned nebulae holds in the fact that:

(i) The contemporary lobes have a larger extent $\left(\sim 20-40^{\prime \prime}\right)$, they are fainter and show less collimation in some cases (see Axis 2). Therefore, the compactness of the young starfish nebulae (with outflow extents less than $\sim 5^{\prime \prime}$ in all cases) and their sharpness of their lobes are lost.

(ii) PC 22 is a high-excitation PN, with dominant [O III] emission, indicating that the central star is hot and the photoionization very efficient. This is contrary to the proto-PNe and young PNe starfish, which were selected for the HST observing program HST-P6353 based on the (very) low [O III $] 5007 \AA$ to $\mathrm{H} \alpha$ line ratio, probing their "early" evolutionary stage. PC 22 would therefore join NGC 6058 (Guillén et al. 2013) in the very small group of evolved starfish PNe.

This investigation opens a new door in the understanding of the history formation and evolution of such objects. The morphology of PC 22 can be explained by a fast and probably primarily uniform wind from the Asymptotic Giant Branch (AGB) phase going through irregular and inhomogeneous cavities from the previous denser AGB shell. Steffen et al. (2013) studied this phenomenon numerically using SHAPE and an adjustable turbulent noise structure (Perlin noise) to simulate, via hydrodynamical modelling, a filamentary AGB shell (their Figure 3) within which a fast wind evolves. By changing the size of the cells or voids of the filamentary sheet, they could reproduce different types of starfish nebulae, indeed showing non symmetric patterns. It resulted, that the smaller the cells, the smaller the resulting outflows and reversely with a loss of collimation (their Figure 4). Steffen et al. (2013) also found that starfish nebulae would only occur if the AGB shell and post-AGB wind keep a rather constant density and velocity with respect to the angular distance from the equator.

PC 22 would therefore be at a stage, still early in the $\sim 10^{4}$ yrs $\mathrm{PN}$ life, where the material dragged by the fast post-AGB wind is going through a more evolved (and larger) AGB shell populated with predominantly wider, randomly distributed and inhomogeneous voids. This therefore generates (mostly) large outflows/lobes, although small structures can also be present. The waist often described in starfish nebulae can therefore be explained as being the condensation of the nebular material at the base of the voids in the filamentary shell. We note that in this scenario, there is no need to invoke a particular launching mechanism or precession as it would be the case in other bipolar or multipolar PNe: the wind-shell interaction, owing the evolving filamentary nature of the latter, seems to work appropriately.

\section{ACKNOWLEDGMENTS}

We would like to thank the reviewer for her/his thorough review and appreciate the comments and suggestions made. LS acknowledges support from PAPIIT grant IA-101316 (Mexico). GR-L acknowl- edges support from CONACYT, CGCI, PRODEP and SEP (Mexico). MAG acknowledges support of the grant AYA 2014-57280-P, co-funded with FEDER funds. SZ acknowledges support from the UNAM-ITE collaboration agreement 1500-479-3-V-04. We thank the daytime and night support staff at the OAN-SPM for facilitating and helping obtain our observations especially to Mr Gustavo Melgoza-Kennedy, our telescope operator, for his assistance during observations, as well as the CATT for time allocation. This article is based upon observations carried out at the Observatorio Astronómico Nacional on the Sierra San Pedro Mártir (OANSPM), Baja California, México. The data presented here were obtained in part with ALFOSC, which is provided by the Instituto de Astrofisica de Andalucia (IAA) under a joint agreement with the University of Copenhagen and NOTSA. We acknowledge the Instituto de Astrofisica de Canarias for the use of the filters. This research has made use of the NASA/IPAC Infrared Science Archive, which is operated by the Jet Propulsion Laboratory, California Institute of Technology, under contract with the National Aeronautics and Space Administration. We have also used archival observations made with the NASA/ESA Hubble Space Telescope, and obtained from the Hubble Legacy Archive, which is a collaboration between the Space Telescope Science Institute (STScI/NASA), the Space Telescope European Coordinating Facility (ST-ECF/ESA) and the Canadian Astronomy Data Centre (CADC/NRC/CSA). IRAF is distributed by the National Optical Astronomy Observatory, which is operated by the Association of Universities for Research in Astronomy (AURA) under a cooperative agreement with the National Science Foundation.

\section{REFERENCES}

Apriamashvili S. P., 1959, Astronomicheskij Tsirkulyar, 202, 13

Barentsen G., Farnhill H. J., Drew J. E., González-Solares E. A., Greimel R., Irwin M. J., Miszalski B., Ruhland C., Groot P., et al., 2014, MNRAS, 444, 3230

Corradi R. L. M., Manso R., Mampaso A., Schwarz H. E., 1996, A\&A, 313, 913

Corradi R. L. M., Sabin L., Miszalski B., Rodríguez-Gil P., SantanderGarcía M., Jones D., Drew J. E., Mampaso A., et al. 2011, MNRAS, 410, 1349

Cuesta L., Phillips J. P., Mampaso A., 1993, A\&A, 267, 199

Fang X., Guerrero M. A., Marquez-Lugo R. A., Toalá J. A., Arthur S. J., Chu Y.-H., Blair W. P., Gruendl R. A., Hamann W.-R., Oskinova L. M., Todt H., 2014, ApJ, 797, 100

Frew D. J., Parker Q. A., Bojičić I. S., 2016, MNRAS, 455, 1459

Giammanco C., Sale S. E., Corradi R. L. M., Barlow M. J., Viironen K., Sabin L., et al. 2011, A\&A, 525, A58

Grosbol P., 1989, in Klare G., ed., Reviews in Modern Astronomy Vol. 2 of Reviews in Modern Astronomy, MIDAS.. pp 242-247

Guerrero M. A., Manchado A., Serra-Ricart M., 1996, ApJ, 456, 651

Guerrero M. A., Miranda L. F., Ramos-Larios G., Vázquez R., 2013, A\&A, 551, A53

Guillén P. F., Vázquez R., Miranda L. F., Zavala S., Contreras M. E., Ayala S., Ortiz-Ambriz A., 2013, MNRAS, 432, 2676

López-Martín L., López J. A., Esteban C., Vázquez R., Raga A., Torrelles J. M., Miranda L. F., Meaburn J., Olguín L., 2002, A\&A, 388, 652

Manchado A., Guerrero M. A., Stanghellini L., Serra-Ricart M., 1996, The IAC morphological catalog of northern Galactic planetary nebulae

Meaburn J., López J. A., Gutiérrez L., Quiróz F., Murillo J. M., Valdéz J., Pedrayez M., 2003, Rev. Mex. Astron. Astrofis., 39, 185

Miszalski B., Corradi R. L. M., Boffin H. M. J., Jones D., Sabin L., Santander-García M., Rodríguez-Gil P., Rubio-Díez M. M., 2011, MNRAS, 413, 1264

Monteiro H., Morisset C., Gruenwald R., Viegas S. M., 2000, ApJ, 537, 853 
Parker Q. A., Acker A., Frew D. J., Hartley M., Peyaud A. E. J., Ochsenbein F., Phillipps S., Russeil D., Beaulieu S. F., Cohen M., Köppen J., Miszalski B., Morgan D. H., Morris R. A. H., Pierce M. J., Vaughan A. E., 2006, MNRAS, 373, 79

Parker Q. A., Bojičić I. S., Frew D. J., 2016, in Journal of Physics Conference Series Vol. 728 of Journal of Physics Conference Series, HASH: the Hong Kong/AAO/Strasbourg $\mathrm{H} \alpha$ planetary nebula database. $\mathrm{p}$. 032008

Peimbert M., Costero R., 1961, Boletin de los Observatorios Tonantzintla y Tacubaya, 3, 33

Rubio G., Vázquez R., Ramos-Larios G., Guerrero M. A., Olguín L., Guillén P. F., Mata H., 2015, MNRAS, 446, 1931

Sabin L., Parker Q. A., Corradi R. L. M., Guzman-Ramirez L., Morris R. A. H., Zijlstra A. A., Bojičić I. S., et al. , 2014, MNRAS, 443, 3388

Sahai R., 2000, ApJ, 537, L43

Sahai R., Morris M. R., Villar G. G., 2011, ApJ, 141, 134

Sahai R., Sánchez Contreras C., Morris M., 2005, ApJ, 620, 948

Soker N., 1997, ApJs, 112, 487

Steffen W., Koning N., Esquivel A., García-Segura G., García-Díaz M. T., López J. A., Magnor M., 2013, MNRAS, 436, 470

Steffen W., Koning N., Wenger S., Morisset C., Magnor M., 2011, IEEE Transactions on Visualization and Computer Graphics, Volume 17, Issue 4, p.454-465, 17, 454

Tajitsu A., Tamura S., 1998, ApJ, 115, 1989

Vázquez R., López-Martín L., Miranda L. F., Esteban C., Torrelles J. M., Arias L., Raga A. C., 2000, A\&A, 357, 1031

Vázquez R., Miranda L. F., Olguín L., Ayala S., Torrelles J. M., Contreras M. E., Guillén P. F., 2008, A\&A, 481, 107

Wilson O. C., 1950, ApJ, 111, 279

This paper has been typeset from a $\mathrm{T}_{\mathrm{E}} \mathrm{X} / \mathrm{L} \mathrm{T} \mathrm{E} \mathrm{X}$ file prepared by the author.

APPENDIX A: OBSERVED AND SYNTHETIC SPECTRA

OF PC 22 


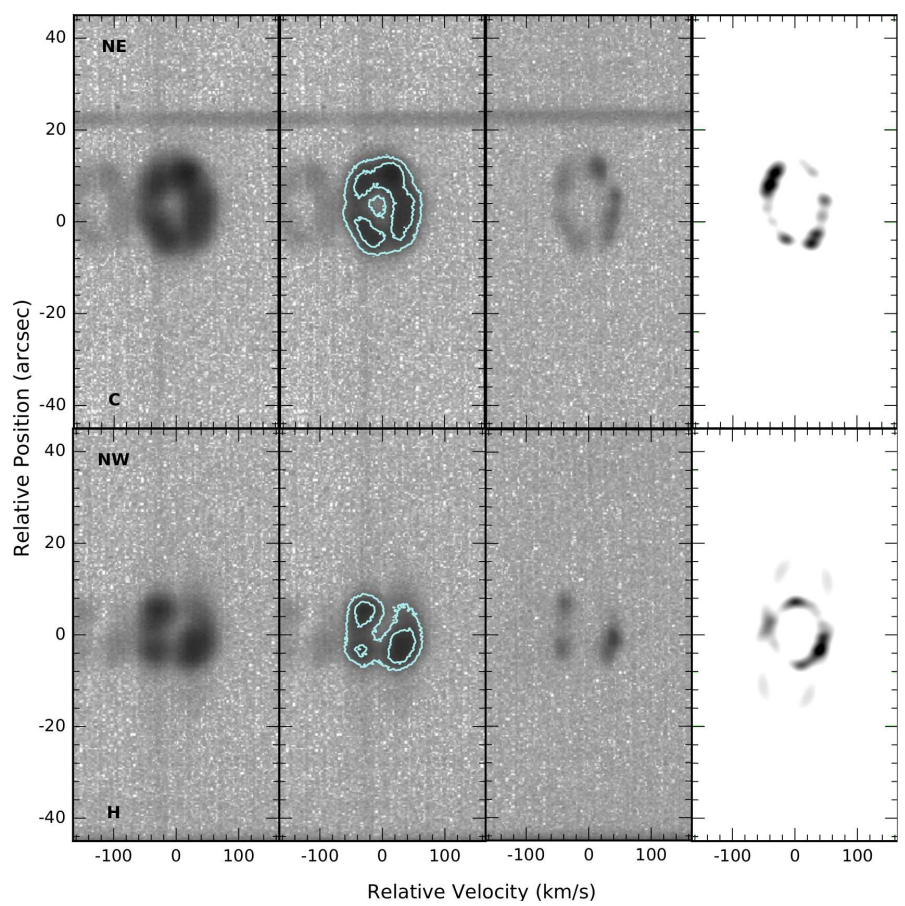

Figure A1. From left to right: MES [O III], [O III]+contours, $\mathrm{H} \alpha$ and [O III] line SHAPE modelling for the slits $\mathrm{C}$ (top) and $\mathrm{H}$ (bottom) 


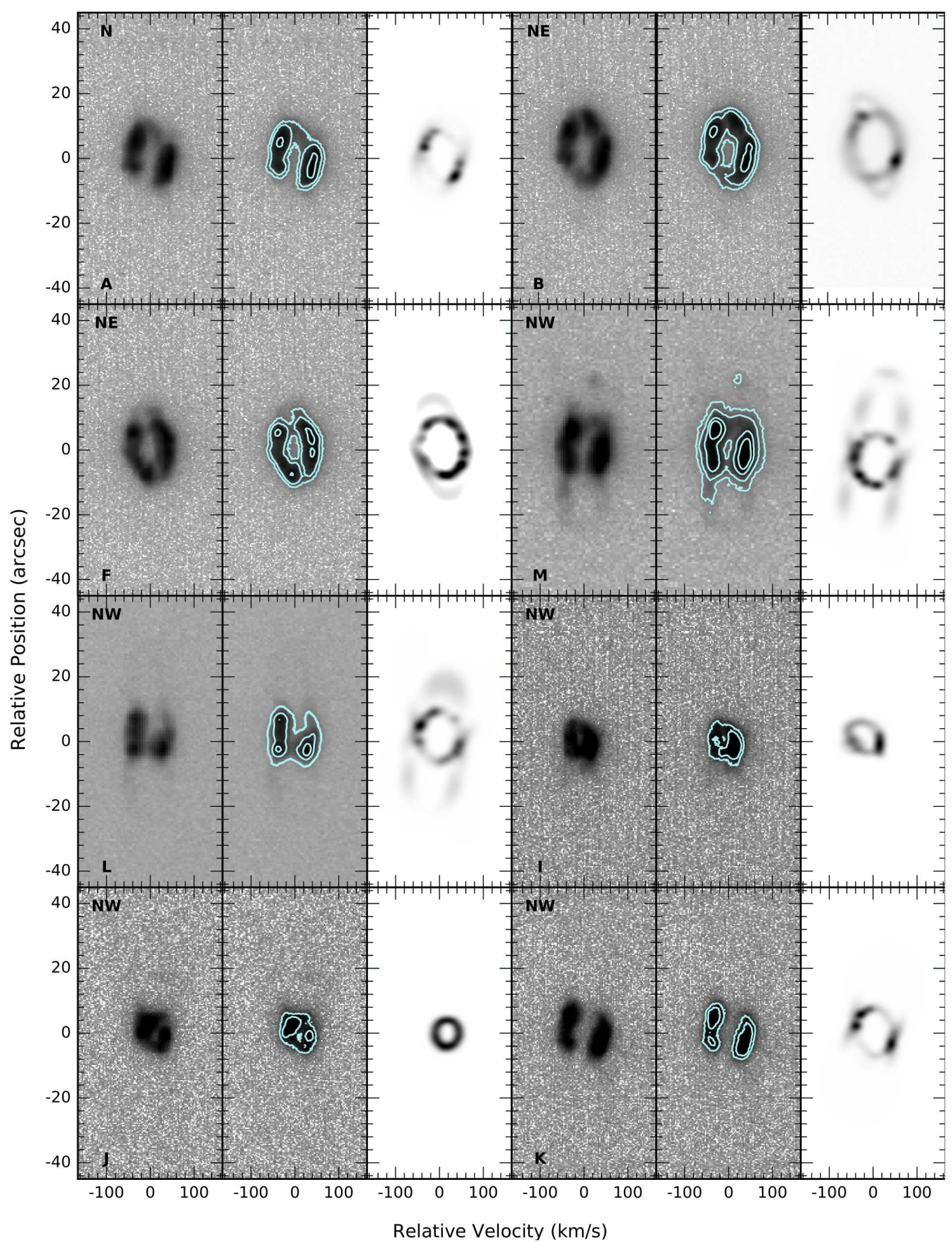

Figure A2. MES [O III], [O III]+contours and [O III] line SHAPE modelling for the slits A,B,F,M,L,I,J and K. 